\title{
Tricuspid Valve Stenosis
}

National Cancer Institute

\section{Source}

National Cancer Institute. Tricuspid Valve Stenosis. NCI Thesaurus. Code C50783.

Narrowing or stricture of the tricuspid orifice of the heart. 